\title{
An EAL domain protein and cyclic AMP contribute to the interaction between the two quorum sensing systems in Escherichia coli
}

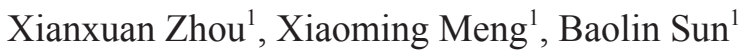 \\ ${ }^{1}$ Hefei National Laboratory for Physical Sciences at Microscale and School of Life Sciences, University of Science and Technology \\ of China, Hefei, Anhui 230027, China
}

Quorum sensing (QS) is a bacterial cell-cell communication process by which bacteria communicate using extracellular signals called autoinducers. Two QS systems have been identified in Escherichia coli K-12, including an intact QS system 2 that is stimulated by the cyclic AMP (cAMP)-cAMP receptor protein (CRP) complex and a partial QS system 1 that consists of SdiA (suppressor of cell division inhibitor) responding to signals generated by other microbial species. The relationship between QS system 1 and system 2 in $E$. coli, however, remains obscure. Here, we show that an EAL domain protein, encoded by $y d i V$, and cAMP are involved in the interaction between the two QS systems in $E$. coli. Expression of $s d i A$ and $y d i V$ is inhibited by glucose. SdiA binds to the $y d i V$ promoter region in a dose-dependent, but nonspecific, manner; extracellular autoinducer 1 from other species stimulates $y d i V$ expression in an sdiA-dependent manner. Furthermore, we discovered that the double sdiA-ydiV mutation, but not the single mutation, causes a 2-fold decrease in intracellular cAMP concentration that leads to the inhibition of QS system 2. These results indicate that signaling pathways that respond to important environmental cues, such as autoinducers and glucose, are linked together for their control in $E$. coli.

Keywords: quorum sensing, $y$ diV , cAMP

Cell Research (2008) 18:937-948. doi: 10.1038/cr.2008.67; published online 17 June 2008

\section{Introduction}

Bacteria have evolved complex genetic circuits to regulate their physiological activities and behaviors in response to a variety of environmental cues. Quorum sensing (QS) is a bacterial cell-cell communication process by which bacterial cells produce, secrete, and detect small-molecule signals named autoinducers, whose concentration corresponds to population density. These extracellular signals include $N$-acylhomoserine lactones (autoinducer-1, AI-1 or AHL) and 4,5-dihydroxy-2,3-pentanedione derivatives (generally called autoinducer-2, AI-2), which serve as signal molecules of QS system 1 and system 2, respectively $[1,2]$. When they reach a threshold concentration in the local environment, a signal transduction cascade

Correspondence: Baolin Sun

Tel: +86-551-3606748; Fax: +86-551-3607438

E-mail:sunb@ustc.edu.cn

Received 23 January 2008; revised 18 March 2008; accepted 24 March 2008; published online 17 June 2008 is initiated, which ultimately results in a coordinated and population-wide alteration in bacterial behaviors such as bioluminescence, exopolysaccharide secretion, biofilm formation, and virulence [2-4].

QS system 1 includes protein pairs similar to LuxR and LuxI. The system 1 autoinducer named AI-1 is produced by LuxI and is detected by LuxR [3]. Two QS systems have been identified in Escherichia coli $\mathrm{K}-12$ [5]. E. coli encodes a LuxR homologue, SdiA (suppressor of cell division inhibitor), but it does not encode a LuxI homolog or synthesize any AHL molecule detected by SdiA [5]. SdiA of E. coli and Salmonella enterica serovar Typhimurium, however, responds to several AHLs generated by other microbial species [6-9]. Recently, a report indicated that E. coli uses SdiA to monitor indole and AHLs to control biofilms; however, it was not ascertained whether indole itself binds to SdiA [10]. A previous study showed that SdiA could support or inhibit RNA polymerase binding to the promoters, thereby affecting the transcription of the target genes [11]. Overexpression of SdiA affects the expression of a battery of genes, including those that control cell 
division, motility, chemotaxis, and multidrug efflux pump genes $[5,6,12,13]$.

E. coli has an intact QS system 2 that includes nine genes (e.g. $l u x S$, $l s r R$, $l s r K$, and $l s r A C D B F G$ ). The cyclic AMP (cAMP)-cAMP receptor protein (CRP) complex stimulates the expression of $l s r R$ and the $l s r$ operon, which includes lsr $A C D B F G$, while LsrR represses their expression and is located adjacent to, while being transcribed divergently from, the $l s r$ operon [14-16]. The system 2 autoinducer named AI- 2 is synthesized by LuxS and accumulates extracellularly. Following internalization by the Lsr transporter encoded by genes in the $l s r$ operon, AI- 2 is phosphorylated by LsrK, and phospho-AI-2 binds specifically to LsrR and antagonizes it. LsrF and LsrG are required for further processing of phospho-AI-2 [17]. It has been recently reported that the mean biofilm thickness and biomass of $l s r R$ or $l s r K$ mutants are lower than those of the wild type, while the global small RNA (sRNA) regulator DsrA and the sRNA cell division inhibitor DicF are induced 2- to 4.4-fold in both $l s r R$ and $l s r K$ mutants [18].

A class of enzymes containing GGDEF domains synthesize the second messenger 3',5'-cyclic diguanylic acid (c-di-GMP) that is later hydrolyzed by EAL or HD-GYP domain proteins [19-23]. In some cases, the GGDEF or EAL domains can play a regulatory role in the absence of a catalytic role; e.g., a catalytically inactive GGDEF domain of the CC3396 protein has retained the ability to bind GTP and, in response, can activate the neighboring EAL domain [24]. As a second messenger in a variety of bacterial species, c-di-GMP influences various aspects of physiology and behavior, such as motility, sessility, virulence, biofilm, and cell-cell communications [25]. The protein encoded by $y d i V$ in $E$. coli, which is $52 \%$ identical at the amino-acid level to its $S$. typhimurium homolog CdgR, consists solely of an EAL domain [26]. In a $c d g R$ mutant of S. typhimurium, the c-di-GMP concentration was about 7-fold higher than that in a wild-type (WT) strain, but CdgR did not exhibit c-di-GMP phosphodiesterase activity in vitro, probably due to lack of suitable trial conditions or indirect regulation of c-di-GMP by CdgR, such as through induction of a phosphodiesterase [27].

Three QS circuits of Vibrio harveyi converge to control one set of genes in a process that involves sRNA species [28, 29]. In E. coli, the cAMP-CRP complex stimulates the expression of both $l s r R$ and the $l s r$ operon by binding to the promoter regions, while LsrR represses their expression $[14,15]$. The relationship between QS system 1 and QS system 2 in E. coli, however, remains obscure. Here, we show that the addition of glucose to the culture inhibited the expression of $s d i A$ and $y d i V$. The expression of $y$ diV was stimulated by SdiA, and extracellular AI-1 from Vibrio fischeri further up-regulated $y d i V$ expression in an $s d i A$-dependent manner. Furthermore, we discovered that $y d i V$ and cAMP contribute to the interaction between the two QS systems in E. coli. On the basis of these results, we propose a working model that describes the signaling pathway linkage in E. coli that includes the two QS systems, cAMP signaling and YdiV.

\section{Results}

Effects of extracellular AI-1 and glucose on ydiV expression

The sequence 5'-AAA AGN NNN NNN NGA AAA-
A

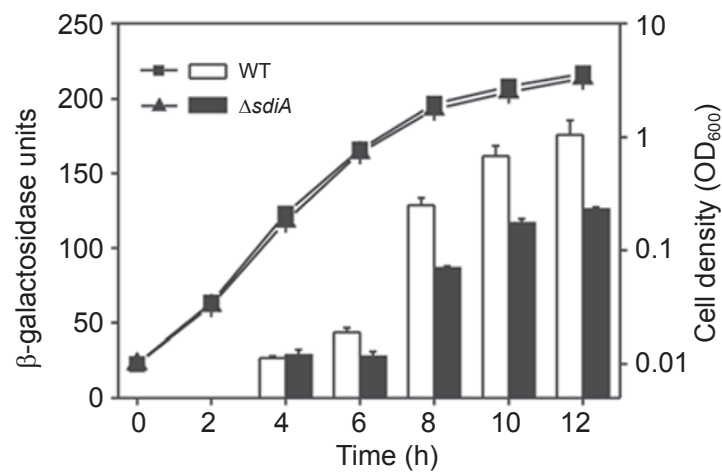

B

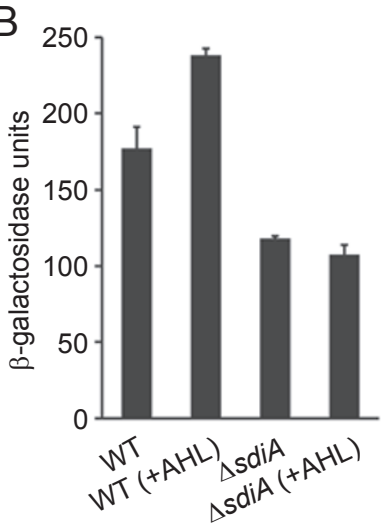

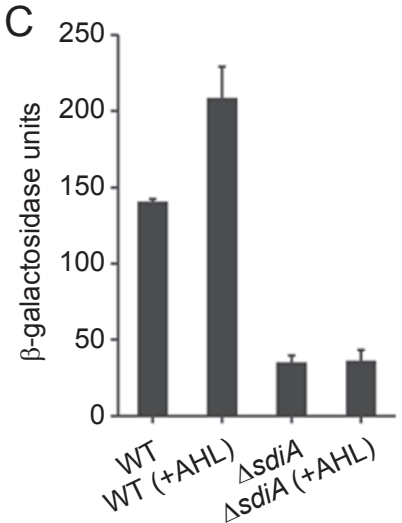

Figure $1 \mathrm{Effects}$ of SdiA and AHL on the expression of ydiV. (A) SdiA stimulates ydiV expression. Conditions for cell growth and $\beta$-galactosidase activity assays are described in Materials and Methods. E. coli ZK126 (WT) and isogenic sdiA mutant carrying plasmid pPydiV (ydiV-lacZ) were grown in LB. At different time points during cell growth, aliquots were collected for the measurement of the $\mathrm{OD}_{600}$ (squares and triangles) and $\beta$-galactosidase activity (bars). (B and $\mathbf{C}$ ) Effects of $\mathrm{AHL}$ on the transcription of ydiV. $\beta$-Galactosidase activity under aerobic conditions (B) and under microaerobic conditions (C) in E. coli ZK126 (WT) and isogenic sdiA mutant carrying plasmid pPydiV (ydiV-lacZ) was measured after growth in LB or LB containing AHL. 
$3^{\prime}$ was identified as the SdiA binding site within the $f t s Q$ promoter region, which is hereafter designated as the SdiA box [11]. Bioinformatics analysis suggested the presence of an SdiA box in the $y d i V$ promoter: $5^{\prime}$-AAA AGg acc cet GAA AA-3' ( -128 to -112 relative to the translation start site of $y d i V$, spacer shown in lowercase letters). A microarray analysis was used in E. coli to identify genes that respond to plasmid-based expression of $s d i A$, and it revealed that the expression of $y d i V$ is elevated with increased sdiA dosage [12]. All of the above imply that extracellular AI-1 might up-regulate $y d i V$ expression in an sdiA-dependent manner.

To investigate whether $y d i V$ is regulated by chromosomal sdiA in vivo, we constructed an sdiA mutant and the $y$ diV-lacZ promoter fusion plasmid $\mathrm{pPydiV}$ and tested $y d i V$ lac $Z$ expression. As indicated by $\beta$-galactosidase activity assays, $s d i A$ deletion lowered the level of $y d i V$ expression by $30 \%$, leaving significant expression of $y d i V$ even in the absence of SdiA (Figure 1A). Several AHLs generated by other microbial species activate SdiA of E. coli and $S$. typhimurium by binding and changing its conformation [6-9]; thus, if chromosomal sdiA up-regulates the expression of $y d i V$, the addition of AHL would elevate $y d i V$ expression. Our results showed that the addition of AHL (referring to oxoC6, a $V$. fischeri AHL signal molecule) resulted in ydi $V$ up-regulation in WT but not in the sdiA mutant, as expected (Figure 1B and 1C). Under aerobic conditions, $y$ diV expression in WT increased 1.5-fold (in Luria-Bertani (LB)) or 2.2-fold (in LB plus AHL) more than that in the sdiA mutant in 12-h-old cultures (Figure 1B). In 24-h-old cultures, the fold-difference for $y d i V$ expression in WT versus the sdiA mutant was not greater than that in 12-h-old cultures under aerobic conditions (data not shown). Under microaerobic conditions, the $y d i V$ expression in WT increased 4-fold (in LB) or 6-fold (in LB plus AHL) more than that in the sdiA mutant in 24 -h-old cultures with $\mathrm{OD}_{600}$ values around 0.57 (Figure 1C). These data demonstrate that AHL from $V$. fischeri further stimulates $y d i V$ expression in WT but not in the sdiA mutant.

To further evaluate whether SdiA can bind to the $y d i V$ promoter region and regulate the expression of $y d i V$, a series of DNA fragments were synthesized, and a gel shift assay was performed with purified SdiA or AHL-SdiA complex. As shown in Figure 2A and 2B, AHL-SdiA complex and SdiA bound the $y d i V$ promoter fragments (S-ydiV) in a dose-dependent manner, and the addition of $10 \mu \mathrm{M}$ AHL to SdiA-binding buffer caused a more intense gel shift (about 20-fold) when using SdiA but not AHL-SdiA complex. The gel shift with AHL-SdiA complex was more intense (about 20-fold) than that with SdiA (Figure 2B). To assess nonspecific binding, we used both the mutated $y$ diV promoter fragments (M-ydiV) and a control DNA (S-con) that is devoid of an SdiA box. The gel shift assay shows that the AHL-SdiA complex bound to M-ydiV, S-ftsQ, and S-con as well (Figure 2A), among which S-ftsQ is the positive control. To exclude any protein contaminant, the purity of SdiA and AHL-SdiA complex preparation was ascertained by SDS-PAGE (Figure 2C). In summary, extracellular AI-1 causes more intense binding of SdiA to the $y d i V$ promoter region, and it up-regulates $y d i V$ expression in an $s$ diA-dependent manner.

To check whether glucose plays an important role in sdiA and $y d i V$ expression, we used the $y d i V$-lacZ promoter fusion plasmid pPydiV described above and constructed the $s$ diA-lacZ promoter fusion plasmid pPsdiA. The addition of $0.8 \%$ glucose to the growth medium decreased the $\beta$-galactosidase activity from the $s d i A$ promoter (2.4-fold) and $y d i V$ promoter (3.6-fold) in WT. $y d i V$ expression in the
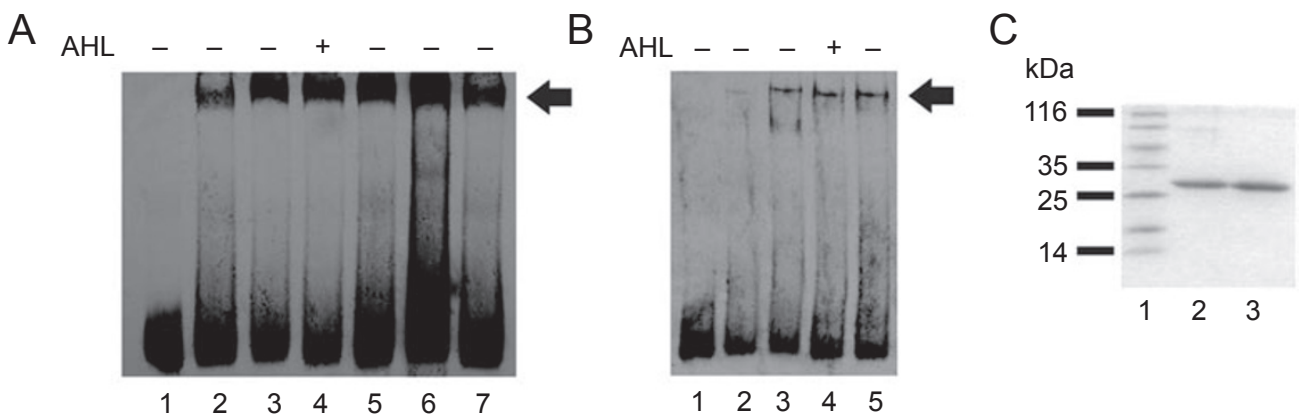

Figure 2 Binding of SdiA to the ydiV promoter. Gel shift assays were performed as described in Materials and Methods. The symbol (+) represents the addition of $10 \mu \mathrm{M}$ AHL to SdiA-binding buffer. The arrow denotes the SdiA-DNA complexes. (A) Gel shift assay was conducted using DNA fragment S-ydiV (lanes 1-4), M-ydiV (lane 5), S-ftsQ (lane 6) and S-con (lane 7) as probes. AHL-SdiA complex was used: lane 1, 0 pmol; lane 2, 7.5 pmol; lanes 3-7, 15 pmol. (B) Gel shift assay was conducted using DNA fragment S-ydiV as probe, SdiA (lane 1, 0 pmol; lanes 2 and 4, 7.5 pmol; lane 3, 15 pmol), and AHL-SdiA complex (lane 5, 7.5 pmol). (C) The purity of SdiA and AHL-SdiA complex was analyzed by SDS-PAGE. Lane 1, protein standard; lane 2, SdiA; lane 3, AHL-SdiA complex. 

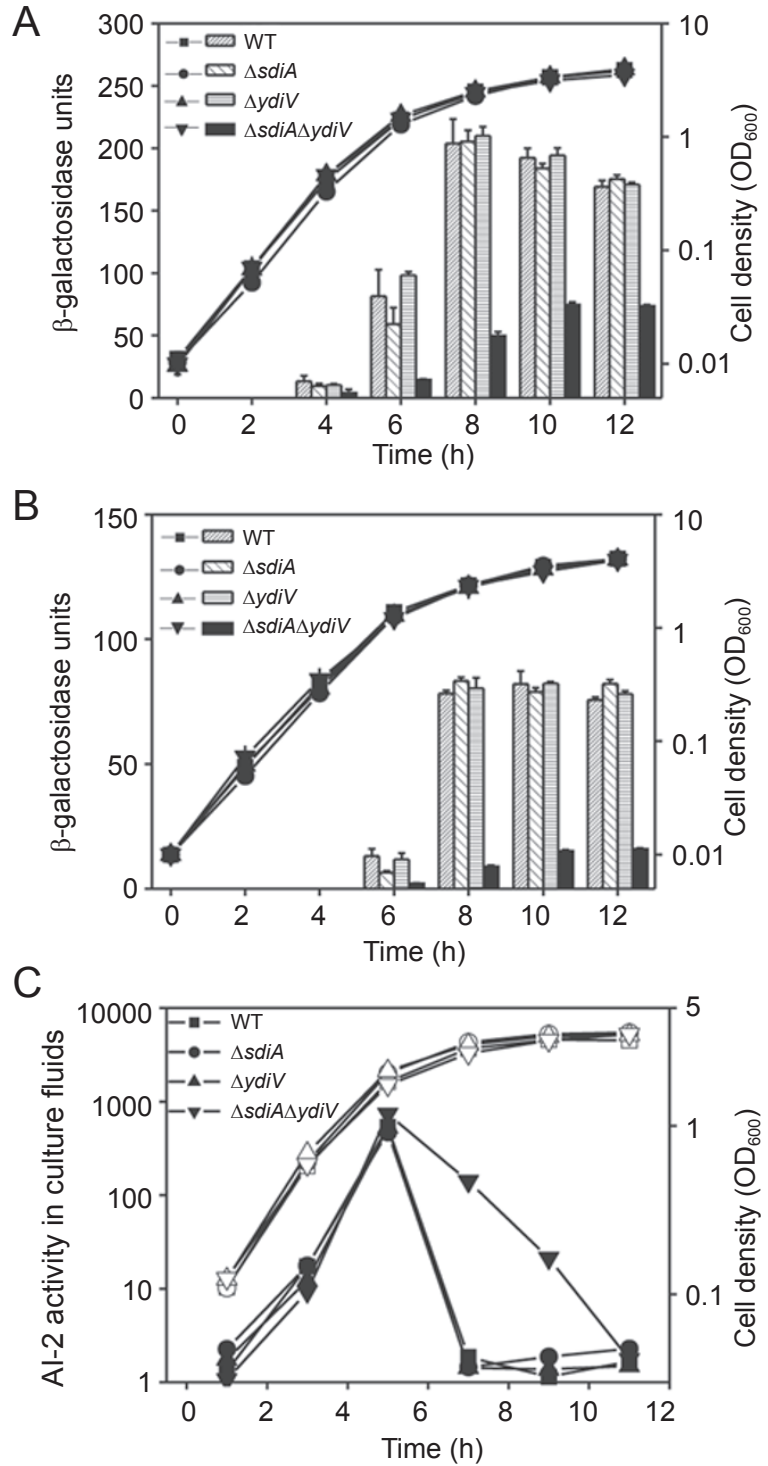

Figure 3 QS system 2 is inhibited in the sdiA-ydiV double mutant. ( $A$ and $\mathbf{B}$ ) The expression of $/ s r R$ and the Isr operon is repressed in the sdiA-ydiV double mutant. E. coli ZK126 (WT), sdiA, ydiV, and sdiA-ydiV double mutant carrying plasmid pPIsrR (IsrR-lacZ) (A) and pPIsrA (Isr-lacZ) (B) were grown in LB. At different time points during cell growth, aliquots were collected for measurement of the $\mathrm{OD}_{600}$ (squares, circles, and triangles) and $\beta$-galactosidase activity (bars). (C) Extracellular Al-2 activity profiles. Overnight cultures of E. coli ZK126 (WT), sdiA, ydiV, and sdiA-ydiV double mutant were diluted 100 -fold into fresh LB medium. At different time points during cell growth, aliquots were collected for measurement of the $\mathrm{OD}_{600}$ (open symbols) and Al-2 activity (solid symbols) as described in Materials and Methods.

sdiA mutant was also down-regulated by the addition of glucose (3.5-fold), while the addition of $10 \mathrm{mM} \mathrm{cAMP} \mathrm{did}$ not restore $\beta$-galactosidase activity to the level observed when the cells were grown in LB alone. These results sug- gest that glucose inhibits $s d i A$ and $y d i V$ expression and that the effect of glucose on $y d i V$ expression is not mediated by SdiA.

The sdiA-ydiV double mutation results in QS system 2 inhibition

In $V$. harveyi, three QS circuits converge to control one set of genes, as mentioned above [28, 29]. However, the interaction between QS systems 1 and 2 in E. coli remains unclear. Together, previous studies and our result show that $y d i V$, sdiA, and QS system 2 are all repressed by glucose $[14,15]$. Some complex processes, namely virulence and biofilm, are regulated by QS system 1, QS system 2, and EAL domain proteins [10, 25, 27, 30-32]. It stands to reason that QS system 1 and system 2 in $E$. coli might be linked and that $y d i V$ might be involved.

$\beta$-Galactosidase activity assays show that $l s r R$ and lsrACDBFG expression was not affected by the deletion of $s d i A$ or $y d i V$, while $s d i A-y d i V$ double mutation resulted in $l s r R$ repression ( $>2.3$-fold) and $l s r A C D B F G$ repression ( $>4.8$-fold) (Figure $3 \mathrm{~A}$ and $3 \mathrm{~B}$ ). As repression of lsr $A C D B F G$ can further affect $\mathrm{AI}-2$ internalization and the subsequent cleavage of phospho-AI-2, we speculated that transport of AI-2 might also be affected in the sdiA-ydiV double mutant. We assayed the extracellular AI-2 profiles of WT and the mutants. Consistent with our results described above, the $s d i A$ or $y d i V$ mutant was indistinguishable from WT in its ability to produce and import AI-2, while the sdiA$y$ diV double mutant showed much slower removal of AI-2 from extracellular fluids compared with that of the other three genotypes when grown in LB at $30{ }^{\circ} \mathrm{C}$ (Figure $3 \mathrm{C}$ ). These results suggest that $y d i V$ plays an important role in the interaction between QS system 1 and system 2, and QS system 2 is repressed by $s d i A-y d i V$ double mutation.

QS system 2 inhibition is caused by decreased intracellular cAMP concentration in the sdiA-ydiV double mutant

In E. coli, AI-2 is synthesized by LuxS and released into the extracellular environment. AI-2 is bound by the periplasmic protein LsrB and internalized by the Lsr transporter (LsrACDB). Following internalization, AI-2 is phosphorylated by LsrK; then, phospho-AI-2 binds specifically to LsrR and antagonizes it. LsrF and LsrG are required for further processing of phospho-AI-2 [14, 17]. The sdiA-ydiV double mutation resulted in QS system 2 inhibition, raising the question of whether this is due to the modulation of a specific factor involved in regulating both $l s r R$ and the $l s r$ operon. The cAMP-CRP complex stimulates the expression of $l s r R$ and the $l s r$ operon, while LsrR represses their expression $[14,15]$, suggesting that cAMP, the CRP, or both may be affected by sdiA-ydiV double mutation. 
To test this hypothesis, $\beta$-galactosidase activity in WT, sdiA mutant, $y d i V$ mutant and $s d i A-y d i V$ double mutant carrying plasmid pPcrp ( $c r p$-lacZ) was determined. The expression of crp was not affected (data not shown). Intracellular cAMP concentration in these strains with different genotypes was also determined. A decreased intracellular cAMP concentration (about 2 -fold, $P<0.05$ ) was observed in the sdiA-ydiV double mutant, while the intracellular cAMP concentration in the sdiA mutant or the $y d i V$ mutant was not significantly different from that in the WT strain (Figure 4A). If the decreased intracellular cAMP concentration in the $s d i A-y d i V$ double mutant caused inhibition of QS system 2, the addition of cAMP should offset the $s d i A-y d i V$ double mutation effects on $l s r R$ and lsrACDBFG expression. To further corroborate our findings, the expression of $l s r R$-lacZ and $l s r$-lacZ in WT and the mutants was measured after $12 \mathrm{~h}$ of growth in LB or LB plus cAMP as described for the experiments shown in Figure $3 \mathrm{~A}$ and 3B. As shown in Figure 4B, the addition of $10 \mathrm{mM}$ cAMP offset the $s$ diA-ydiV double mutation effects on $l s r R$ and $l s r A C D B F G$ expression. These data allowed us to conclude that sdiA and $y d i V$ play a synergistic role in the regulation of intracellular cAMP, and a decrease in the intracellular cAMP concentration caused by the $s d i A-y d i V$ double mutation leads to down-regulation of $l s r R$ and the $l s r$ operon. Hence, cAMP and $y d i V$ are crucial in connecting QS system 1 and QS system 2 in E. coli.

\section{Discussion}

Previous studies have shown that an unidentified extracellular factor in E. coli conditioned medium down-regulated expression of the $s d i A$ gene that normally functions to stimulate $f t s Q \mathrm{P} 2$ expression [33]. The addition of indole up-regulated expression of sdiA (2.9-fold) and led to a decrease in $f t s Q \mathrm{P} 2$ expression [10], so further investigation is needed to determine whether the unidentified extracellular factor, which was shown to regulate $s d i A$ and to regulate the fts $Q 2 p$ cell division promoter via SdiA, is indole. The $s$ diA gene was not affected by luxS deletion in LB with or without glucose [15], and our study showed that deletion of $l s r R$ did not affect $s d i A$ expression in LB (data not shown). Thus, the extracellular factor is unlikely to be AI-2, and AI-2 does not signal to SdiA in these experimental conditions.

A previous study showed that $S$. typhimurium SdiA, which is $69 \%$ identical at the amino-acid level to its $E$. coli homolog, could partially complement the E. coli sdiA gene for activation of fts $Q A Z$ at promoter 2 and for suppression of filamentation caused by an ftsZ(Ts) allele [34]. In S. typhimurium, overexpressed plasmid-based $s d i A$, but not chromosomal $s d i A$, can activate four genes, $\operatorname{srg} A, \operatorname{srg} B$, $r c k$, and $\operatorname{srg} C$ [34]. Microarray analysis revealed that more
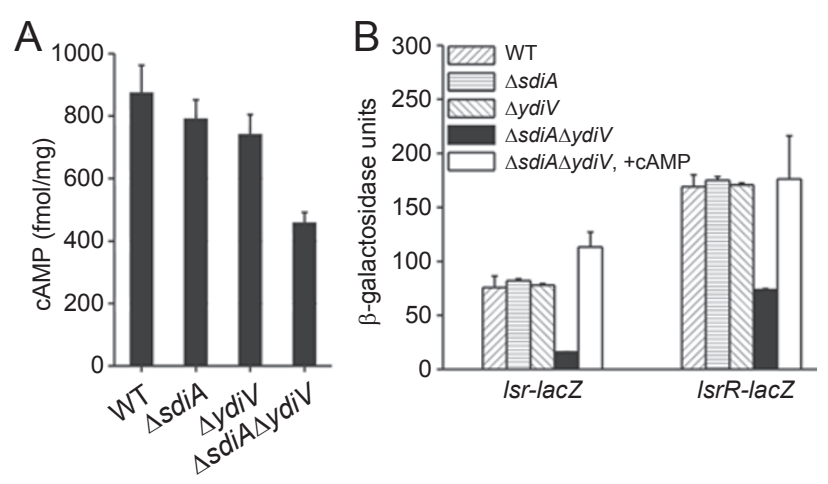

Figure 4 QS system 2 inhibition is caused by a decreased intracellular cAMP concentration in the sdiA-ydiV double mutant. (A) Intracellular cAMP concentration determination. Intracellular CAMP concentrations were measured by the cAMP enzyme immunoassay system and expressed in femtomoles per milligram (wet weight) of cells. The $P$ value for the sdiA-ydiV double mutation was <0.05. (B) The sdiA-ydiV double mutation effects on IsrR and Isr operon expression are offset by the addition of cAMP. Strains were grown in LB or LB plus $10 \mathrm{mM}$ cAMP. $\beta$-Galactosidase activity in strains carrying plasmid pPIsrA (Isr-lacZ) or pPIsrR (IsrR-lacZ) was measured after $12 \mathrm{~h}$ of growth.

than 100 genes are regulated by $s d i A$ overexpression, and the expression of $y d i V$ was elevated with increased $s d i A$ dosage [12]. However, the genes identified with the microarray were not tested for a response to chromosomal sdiA or AHL. In the present study, we found that $s d i A$ deletion lowers the level of $y$ di $V$ expression, and AHL from $V$. fischeri further stimulates $y d i V$ expression in WT but not in the sdiA mutant. Our results also show that, under microaerobic conditions, the fold-difference for $y d i V$ expression in WT versus $S d i A$ mutant is higher than that under aerobic conditions. This result suggests that there might be an interaction between respiratory control and $y d i V$ expression. $E$. coli sdiA plays a more important role under microaerobic conditions and may be used to detect the transition from a free-living state to the intestinal environment [34].

As shown by the gel shift assay, we found that the addition of AHL to SdiA-binding buffer caused more intense binding of SdiA to the $y d i V$ promoter region. The addition of AHL to SdiA-binding buffer did not cause more intense binding of the AHL-SdiA complex to the $y$ diV promoter region because the SdiA binding pocket had been occupied by AHL during induction. The gel shift with the AHL-SdiA complex was more intense than that with SdiA. These results suggest that AHL supports SdiA binding to the $y d i V$ promoter. Our results further corroborate the previously stated hypothesis that SdiA has very low levels of activity in the absence of AHL and that the addition of AHL leads to a more stable folded protein $[5,8,35]$. So far, the binding 
specificity of SdiA is still controversial. The SdiA binding site within the $f t s Q \mathrm{P} 2$ promoter, which is designated as the SdiA box, was identified previously [11]. However, this SdiA box is not present in the promoters of any SdiAregulated genes of Salmonella, including the Salmonella $\operatorname{srg} E$-lux fusion that was placed into $E$. coli $\mathrm{K}-12$ and was activated in an $s d i A$-dependent and AHL-dependent manner [5]. A DNA fragment ( $73 \mathrm{bp}$ from +112 to +184 with respect to the fts $Q \mathrm{P} 2$ initiation site) formed complexes with SdiA as shown by the gel shift assay, but clear protection was not observed in this region as shown by DNase I footprinting, and there was no homolog of SdiA box present in this DNA fragment [11]. To assess nonspecific binding, we used both the mutated $y d i V$ promoter fragment (M-ydiV) and a control DNA (S-con) that is devoid of an SdiA box. Our results show that the AHL-SdiA complex, as well as SdiA (data not shown), can bind to the $y d i V$ promoter fragments (S-ydiV), the $y d i V$ promoter fragments containing base-substitutions in the SdiA box (M-ydiV), the $f t s Q P 2$ fragments (S-ftsQ) as a positive control, and a 44 bp DNA fragment of the multicloning site of pBluescript II KS+ (S-con) (Figure 2A). The negative control DNA (S-con) displayed reduced binding as compared to $y d i V$ promoter fragments (Figure $2 A)$. The results from the SDS-PAGE analysis indicate that there were no protein contaminants in the SdiA and AHL-SdiA complex preparations (Figure 2C). As shown in Figure 2A and 2B, the gel shift is very weak even with 7.5 pmol of SdiA, a result that is consistent with a previous study [11]. There is no apparent difference between the specific $y d i V$ promoter and the mutated $y d i V$ promoter when the gel shift assay was conducted with 7-28 pmol of SdiA (data not shown). Our results show that, whether or not AHL is present, SdiA binds DNA nonspecifically, at least in vitro in our experimental conditions. Because the action of SdiA is pleiotropic, one would expect that other physicochemical factors or proteins might be involved in the control of binding specificity of SdiA in vivo.

Although the evidence for indirect interplay between QS and c-di-GMP signaling is accumulating, a direct connection, such as the activation of a c-di-GMP synthetase or phosphodiesterase by an autoinducer, has yet to be reported [36-38]. Besides YdiV (EAL), a GGDEF domain protein YedQ was also up-regulated with increased sdiA dosage [12]. These results indicate that SdiA might play an important role in GGDEF/EAL-related cellular processes. Lee et al. [10] recently reported that AHLs inhibited $E$. coli biofilm formation through SdiA in a dose-dependent manner and that $s d i A$ mutation caused a 51-fold increase in biofilm formation. Since biofilm formation is suppressed upon overproduction of EAL domain proteins [25, 39], the SdiA-dependent expression of $y$ diV (EAL) might also contribute to the SdiA-mediated inhibition of biofilm for- mation in E. coli.

Interestingly, E. coli has 19 GGDEF and 17 EAL domain proteins, with an overlap of seven proteins containing both domains. Temporal or spatial regulatory mechanisms might be employed by a single GGDEF or EAL protein to significantly affect a certain function [40]. Although the function of these $29 \mathrm{GGDEF} / \mathrm{EAL}$ domain proteins in $E$. coli is poorly understood, it is obvious that they play a key role in c-di-GMP signaling $[40,41]$. So far, it is not clear whether YdiV in E. coli acts as a phosphodiesterase or just plays a regulatory role. Here, we have shown that YdiV is tightly connected with QS systems and cAMP signaling in E. coli.

The cAMP-CRP complex stimulates expression of both $l s r R$ and the $l s r$ operon of $E$. coli, which are divergently transcribed, by directly binding to the two different CRP binding sites in the intergenic region $[14,15]$. CRP adopts an active conformation only when it binds CAMP, and the concentration of the active CAMP-CRP complex is influenced by the intracellular concentration of cAMP [42]. In the present study, we found that the intracellular cAMP concentration, but not the expression of $c r p$, was decreased by about 2-fold in the sdiA-ydiV mutant, and the decreased cAMP concentration subsequently led to inhibition of expression of $l s r R$ and the $l s r$ operon. In a later trial, the addition of cAMP offset the $s d i A-y d i V$ double mutation effects on $l s r R$ and $l s r A C D B F G$ expression. These results raise the question of how the decreased intracellular concentration of cAMP could be caused by the sdiA-ydiV double mutation.

E. coli strains employ a complex mechanism to control intracellular cAMP concentration, and, so far, this process has not been completely elucidated. The intracellular cAMP concentration in E. coli can be controlled by its own synthesis and hydrolysis [42]. cAMP is synthesized by adenylate cyclase (AC) which is encoded by the cyaA gene, and the activity of $\mathrm{AC}$ is regulated transcriptionally and post-translationally [42]. The transcription of $c y a A$ is negatively regulated by the cAMP-CRP complex; thus, a large increase in cAMP synthesis occurs in a crp mutant [43]. However, the cellular levels of cAMP are regulated mainly by post-translationally modified IIA ${ }^{\text {Glc }}[43,44]$. The phosphorylated form of IIA ${ }^{\mathrm{Glc}}$, of the phosphoenolpyruvate: sugar phosphotransferase system (PTS), binds to AC and stimulates its activity only in the presence of additional unknown factor(s) from E. coli extracts [44]. The hydrolysis of cAMP by endogenous cAMP phosphodiesterases provides another way to affect the intracellular cAMP concentration. In E. coli, cAMP is hydrolyzed by CpdA [45]. In the present study, we have discovered that the EAL domain protein encoded by $y d i V$ is involved in the regulation of cAMP production and that it plays an important role in the 
interaction between QS system 1 and QS system 2 in $E$. coli. As shown in Figure 4A, the intracellular concentration of cAMP is decreased in the $s d i A-y d i V$ mutant than that in the $y d i V$ mutant, indicating that SdiA positively regulates cAMP production in the mutants with an $y d i V$ deletion, as does YdiV in the mutants with an sdiA deletion. It is possible that SdiA and YdiV synergistically regulate the intracellular concentration of cAMP to control QS system 2 in $E$. coli. The factors involved in cAMP production, such as $c y a A$, IIA ${ }^{\mathrm{Glc}}$, or CpdA, might be affected by $s d i A-y d i V$ double mutation. Identification of the exact mechanism of decreased intracellular cAMP concentration in the sdiA$y d i V$ double mutant will be the subject of further studies.

In the present study, we investigated the environmental cues and regulatory pathway interactions that influence the E. coli expression profile and physiological activities. As shown in Figure 5, QS system 1 and QS system 2 in E. coli are linked via the EAL domain protein encoded by $y d i V$ and cAMP, and in this process, unidentified $\mathrm{X}$ factor(s), possibly cyaA, IIA ${ }^{\mathrm{Glc}}$, or CpdA, might be involved. When glucose is present in the growth medium, the expression of $l s r R$ and the $l s r$ operon is apparently repressed, and the level of $l u x S$ transcription increases $[14,15]$. Here, we found that SdiA, a LuxR homolog, and the EAL domain protein encoded by $y d i V$ were also repressed at the transcriptional level by the addition of glucose and that the effect of glucose on $y \mathrm{diV}$ expression was not mediated by SdiA. These results suggest that QS systems and YdiV in E. coli mainly function under specific growth conditions, especially during a shortage of glucose or other PTS sugars. The expression of $y d i V$ is upregulated by QS system 1, and it contributes to the control of intracellular cAMP concentration, which leads to the inhibition of QS system 2 in the sdiA-ydiV double mutant. Interestingly, $l s r R$ and $l s r B$ of $E$. coli are repressed (4- to 5 -fold) under high levels of c-di-GMP in cells overexpressing $y d d V$, a diguanylate cyclase [46]. Thus, it is clear that E. coli cells have complex hierarchical regulatory systems and that these important signaling pathways, including QS systems, cAMP, and GGDEF/EAL domain proteins, are linked together for their control. Identification of the mechanisms employed by the $s d i A-y d i V$ double mutant to control intracellular cAMP concentration will enable us to address the different roles of $s d i A$ and $y d i V$ during this process and to clarify further intracellular and intercellular signaling in E. coli.

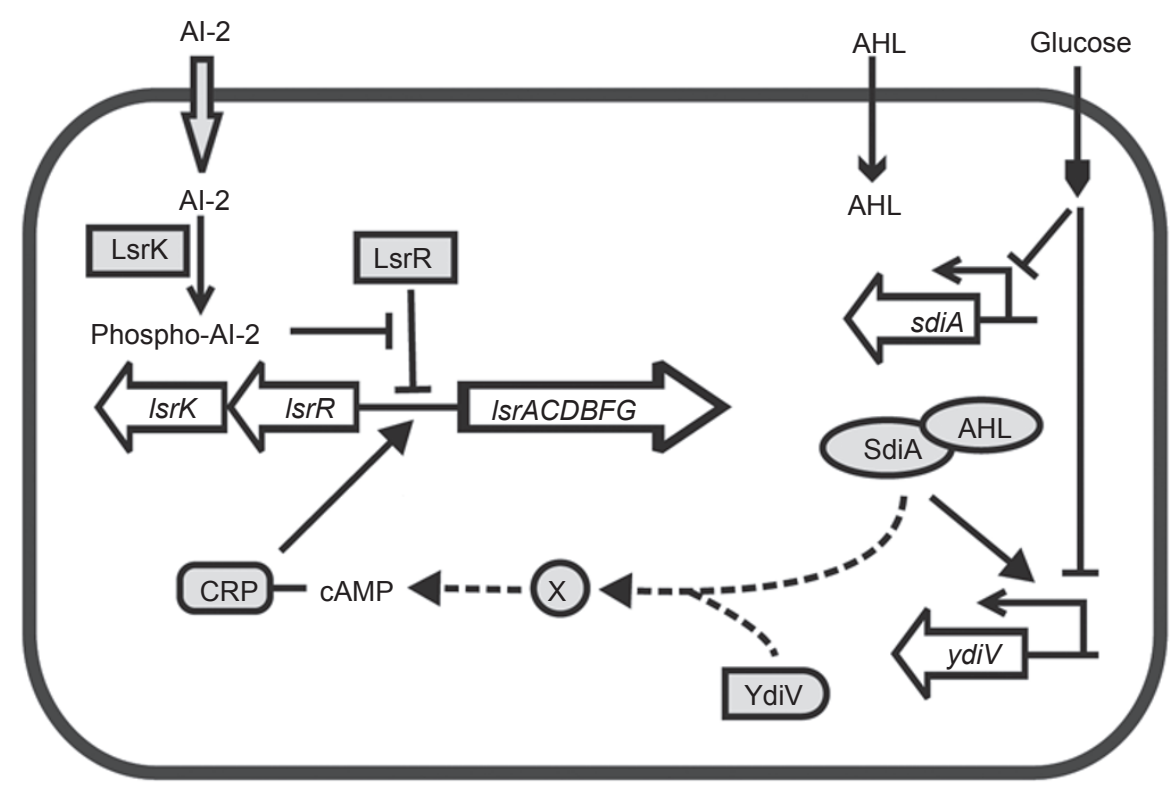

Figure 5 Conceptual model of signaling pathway interactions in E. coli. Al-2 is internalized by the Lsr transporter encoded by IsrACDB and phosphorylated by LsrK. Phospho-Al-2 binds specifically to LsrR and antagonizes it. Expression of IsrR and the Isr operon is inhibited by LsrR and stimulated by the CAMP-CRP complex. LsrF and LsrG are required for further processing of phospho-Al-2. Besides the inhibition effect on QS system 2, glucose represses the expression of sdiA and ydiV. The expression of $y d i V$ is stimulated by the AHL-SdiA complex, and it contributes to the control of intracellular cAMP concentration, which leads to the inhibition of QS system 2. Regulatory effects are shown by solid (direct) or dashed lines (indirect or unclear mechanisms). Positive and negative regulatory effects are indicated by lines with triangular arrowheads and by lines with blunt ends, respectively. 


\section{Materials and Methods}

\section{Bacterial strains, plasmids and media}

The bacterial strains and plasmids used in this work are listed in Table 1. E. coli ZK126 was used as WT strain [47]. Bacteria were grown in LB medium or on LB plates containing 1.5\% agar under aerobic conditions unless otherwise indicated. The autoinducer bioassay $(\mathrm{AB})$ and Luria-marine (LM) media are described in detail elsewhere [48]. Unless otherwise stated, glucose, cAMP, and $\mathrm{N}$-(3oxohexanoyl)-homoserine lactone (oxoC6, which serves as a $V$. fischeri AHL signal molecule) were added at $0.8 \%, 10 \mathrm{mM}$, and $10 \mu \mathrm{M}$, respectively. AHL was added to media as dilutions from a $10 \mathrm{mM}$ stock solution in ethyl acetate. The final concentration of ethyl acetate was $0.1 \%$ and had no effect on the growth or AHL response of the reporter. Ampicillin (Ap) and kanamycin (Kan) were used respectively at 100 and $50 \mu \mathrm{g} / \mathrm{ml}$ when necessary.

\section{Plasmid construction}

The plasmids used in this study are listed in Table 1 and standard procedures were employed [49]. Genes and promoters were prepared by PCR using $E$. coli ZK126 chromosomal DNA as template. Extractions of DNA from agarose gels were performed using Wizard ${ }^{\circledR} \mathrm{PCR}$ Preps DNA Purification System (Promega, Madison, WI). Primers used in this study are listed in Table 2. All constructs made by PCR were sequenced to verify their integrity.
Plasmid pFZY1, which was used to construct promoter fusion vectors in this study, is a low-copy-number vector (one to two copies per cell) with the multiple restriction site linker of M13mp18 inserted upstream from a promoterless galK'-lacZYA reporter segment [50]. To create pPydiV (ydiV-lacZ), pPsdiA (sdiA-lacZ), and pPcrp (crplacZ), the promoter regions were amplified by PCR using the primers P-ydiV-F/P-ydiV-R, P-sdiA-F/P-sdiA-R, and P-crp-F/P-crp-R, respectively. The purified PCR products were digested with appropriate restriction enzymes, as shown in Table 2, and ligated into digested pFZY 1. Plasmids pPlsrR (lsrR-lacZ) and pPlsrA (lsr-lacZ) were constructed by amplification of the same promoter region using primers P-lsr-R and P-lsr-A followed by purification, digestion with EcoRI and insertion into EcoRI-digested and dephosphorylated pFZY1, while the transcriptional direction was determined by PCR using primers P-1sr-R (or P-lsr-A) and Mp18-R that anneals to pFZY1.

To express a His-tagged version of SdiA, pGsdiA was constructed. DNA fragments containing the sdiA coding region were amplified by PCR using primers G-sdiA-F/G-sdiA-R. After digestion with the appropriate restriction enzymes as shown in Table 2, the amplified DNA fragments were cloned into the corresponding sites of pET-28a $(+)$. pGsdiA expresses SdiA with a N-terminal hexahistidine tag.

\section{Gene disruption}

Chromosomal deletions of $s d i A, y d i V$, and $l s r R$ were constructed by methods described previously [51]. We used the phage $\lambda$ Red

Table 1 Bacterial strains and plasmids used in this study

\begin{tabular}{|c|c|c|}
\hline Strain or plasmid & Relevant genotype or characteristics & Source or reference \\
\hline \multicolumn{3}{|l|}{ E. coli strains } \\
\hline ZK126 & W3110 $\Delta l a c$ tna-2 & [47] \\
\hline $\mathrm{SCH} 603$ & ZK126 $\Delta$ sdiA::Kan & This study \\
\hline SCH604L & ZK126 $\Delta l s r R::$ Kan & This study \\
\hline SCH604S & $\mathrm{SCH} 603 \Delta s d i A$ & This study \\
\hline SCH604SB & $\mathrm{SCH} 604 \mathrm{~S} \Delta s d i A \Delta y d i V:: \mathrm{Kan}$ & This study \\
\hline SCH604B & ZK126 $\Delta y d i V::$ Kan & This study \\
\hline \multicolumn{3}{|l|}{ Plasmids } \\
\hline pET-28a $(+)$ & Expression vector, $\operatorname{Kan}^{\mathrm{R}}$ & Novagen \\
\hline pFZY1 & $\begin{array}{l}\text { galK'-lacZYA transcriptional } \\
\text { fusion vector, } \mathrm{Ap}^{\mathrm{R}}\end{array}$ & {$[50]$} \\
\hline pGsdiA & $\begin{array}{l}\text { pET-28a }(+) \text { carrying } s d i A \text { of } \\
\text { E. coli } \mathrm{Kan}^{\mathrm{R}}\end{array}$ & This study \\
\hline pPsdiA & $\begin{array}{l}\mathrm{pFZY} 1 \text { derivative, containing } \\
\text { sdiA promoter region, } \mathrm{Ap}^{\mathrm{R}}\end{array}$ & This study \\
\hline pPlsrR & $\begin{array}{l}\text { pFZY1 derivative, containing } l s r R \\
\text { promoter region, } \mathrm{Ap}^{\mathrm{R}}\end{array}$ & This study \\
\hline pPlsrA & $\begin{array}{l}\text { pFZY1 derivative, containing } l s r A \\
\text { promoter region, } \mathrm{Ap}^{\mathrm{R}}\end{array}$ & This study \\
\hline pPydiV & $\begin{array}{l}\mathrm{pFZY} 1 \text { derivative, containing } y d i V \\
\text { promoter region, } \mathrm{Ap}^{\mathrm{R}}\end{array}$ & This study \\
\hline pPcrp & $\begin{array}{l}\text { pFZY1 derivative, containing } c r p \\
\text { promoter region, } \mathrm{Ap}^{\mathrm{R}}\end{array}$ & This study \\
\hline
\end{tabular}


Table 2 Oligonucleotides used in this study

\begin{tabular}{|c|c|}
\hline Name & Sequence $\mathrm{e}^{\mathrm{a}, \mathrm{b}, \mathrm{c}, \mathrm{d}}$ \\
\hline G-sdiA-F & GTCATGccatggGACACCACCACCACCACCACCAGGATAAGGATTTTTTCAGCTGGC \\
\hline G-sdiA-R & GATCCgaattcTTAAATTAAGCCAGTAGCGGCCGCGTAACAG \\
\hline P-lsr-A & CGCgaattcTCGATGCCTTTCAGGACATT \\
\hline Mp18-R & AAGCTTGCATGCCTGCAGGTC \\
\hline P-ydiV-F & CCGgaattcCTACCAGCAAGGGAGTGATGC \\
\hline P-ydiV-R & AGAggatccTAATTAACTCAACGCCGACAA \\
\hline P-crp-F & CCGgaattcGAAAAGTTAACCCTTCGACCCA \\
\hline P-crp-R & AGAggatccTGGCAATGAGACAAGAACCAT \\
\hline D-ydiV-R & TTATCGCTGAACCAACGTCGTTATCTGATTTATGGGGTGGGCGAAGAACTCCAGCATGA \\
\hline D-lsrR-F & ATGACAATCAACGATTCGGCAATTTCAGAACAGGGAATGTAGGCTGGAGCTGCTTCG \\
\hline D-lsrR-R & TTAACTACGTAAAATCGCCGCTGCTGTGTCCTGATCGATGGGAATTAGCCATGGTCC \\
\hline S-ydiV-F & TCAGGATAATCTAAAAAAAGGACCCCTGAAAAAGTGGTTAACA \\
\hline S-ydiV-R & TCCATGTTAACCACTTTTTCAGGGGTCCTTTTTTTAGATTATC \\
\hline M-ydiV-F & TCAGGATAATCTAAAcAtAGGACCCCTGActAAGTGGTTAACA \\
\hline M-ydiV-R & TCCATGTTAACCACTTagTCAGGGGTCCTaTgTTTAGATTATC \\
\hline S-ftsQ-F & CAGTGTGGGAATGTCAAAAGTAGTAGCAGAAAATGCTCTACAAG \\
\hline S-ftsQ-R & GCATCTTGTAGAGCATTTTCTGCTACTACTTTTGACATTCCCAC \\
\hline
\end{tabular}

${ }^{a}$ The underlined sequences anneal to the template plasmid, while the remaining sequences correspond to the ends of the indicated genes.

${ }^{\mathrm{b}}$ The boxed-in sequences represent the potential SdiA box.

${ }^{\mathrm{c}}$ The sequences in bold lowercase letters refer to the restriction endonuclease recognition sites. NcoI (ccatgg), EcoRI (gaattc), XhoI (ctcgag), Bam HI (ggatcc), KpnI (ggtacc).

${ }^{\mathrm{d}}$ The base substitutions in the SdiA box of M-ydiV-F or M-ydiV-R are shown in lowercase letters.

recombination system to replace $s d i A$ with an $s d i A::$ Kan fragment, which was amplified by PCR from the plasmid pKD4 using primers D-sdiA-F/D-sdiA-R (Table 2). The resulting PCR product was treated with $D p n I$, purified and introduced by electroporation into E. coli ZK126 carrying the Red recombinase expression plasmid pKD46, which was cured later by growth at $42{ }^{\circ} \mathrm{C}$. Recombinants were selected on LB supplemented with kanamycin. Deletions of $y d i V$ and $l s r R$ were constructed similarly by PCR amplification of pKD4 with primers D-ydiV-F/D-ydiV-R and D-lsrR-F/D-lsrR-R, respectively. To construct strain $\mathrm{SCH} 604 \mathrm{~S}$, the $\mathrm{Kan}^{\mathrm{R}}$ gene of strain SCH603 was eliminated using the previously described method, and pCP20 was employed [51]. Strain SCH604SB was constructed similarly by introducing an $y d i V::$ Kan fragment into E. coli SCH604S containing plasmid pKD46. All of the gene deletions were verified by PCR tests.

\section{Protein purification}

His-tagged SdiA was prepared as described previously with some modifications $[11,35]$. Transformants of $E$. coli BL21(DE3) containing pGsdiA were grown in LB to an optical density of 0.6 at $600 \mathrm{~nm}$, induced with $0.8 \mathrm{mM}$ IPTG, or induced with $0.8 \mathrm{mM} \mathrm{IPTG}$ as well as supplied with $100 \mu \mathrm{M}$ AHL to form AHL-SdiA complex during induction with IPTG and then incubated for an additional 3 h. All subsequent procedures were performed at $4{ }^{\circ} \mathrm{C}$. The cultures were harvested and resuspended in $40 \mathrm{ml}$ of buffer A (20 mM Tris$\mathrm{HCl}, \mathrm{pH} 8.0$, at $4{ }^{\circ} \mathrm{C} ; 500 \mathrm{mM} \mathrm{NaCl} ; 5 \mathrm{mM}$ 2-mercaptoethanol and $5 \%$ glycerol $)$. After the addition of PMSF ( $1 \mathrm{mM})$ and lysozyme $(0.1 \mathrm{mg} / \mathrm{ml})$, the cells were incubated on ice for $30 \mathrm{~min}$ and then lysed by sonication. After centrifugation at $16000 \mathrm{rpm}$ for $30 \mathrm{~min}$, the supernatant was applied to a nickel-NTA column and eluted with a gradient of 20-250 mM imidazole in buffer A. Eluted fractions were subjected to SDS-PAGE analysis. Fractions containing the overexpressed His-tagged SdiA were pooled and dialyzed against SdiA-dialysis buffer $\left(20 \mathrm{mM}\right.$ Tris- $\mathrm{HCl}, \mathrm{pH} 7.6$, at $4{ }^{\circ} \mathrm{C} ; 200 \mathrm{mM}$ $\mathrm{KCl} ; 10 \mathrm{mM} \mathrm{MgCl}_{2} ; 0.1 \mathrm{mM}$ EDTA; $1 \mathrm{mM}$ DTT and 50\% glycerol) and stored at $-80{ }^{\circ} \mathrm{C}$ until use. The purity of SdiA and AHL-SdiA 
complex was analyzed by SDS-PAGE.

\section{Gel shift assay}

Double-stranded DNA fragments including the $y d i V$ promoter fragments (S-ydiV, S-ydiV-F/S-ydiV-R), the $y d i V$ promoter fragments containing base-substitutions in the SdiA box (M-ydiV, M-ydiV-F/MydiV-R), the fts $Q$ promoter fragments (S-ftsQ, S-ftsQ-F/S-ftsQ-R), and a $44 \mathrm{bp}$ DNA fragment of the multicloning site of pBluescript II KS+ (S-con, S-con-F/S-con-R) were produced by boiling and slowly cooling synthetic DNA oligonucleotides (Table 2). The DIG gel shift kit (Roche Ltd., Mannheim, Germany) was used for the labeling of DNA fragments and the detection of signals according to the manufacturer's instructions. Band intensities were analyzed with Stratagene EagleSight software.

The labeled DNA fragments (75 fmol) were incubated with various amounts of purified SdiA or AHL-SdiA complex at $4{ }^{\circ} \mathrm{C}$ for $30 \mathrm{~min}$ in $10 \mu \mathrm{l}$ of SdiA-binding buffer $\left(20 \mathrm{mM}\right.$ Tris-HCl, $\mathrm{pH} 8.0$, at $4{ }^{\circ} \mathrm{C}$; $50 \mathrm{mM} \mathrm{NaCl} ; 3 \mathrm{mM}$ magnesium acetate; $0.1 \mathrm{mM}$ EDTA; $0.1 \mathrm{mM}$ DTT and $25 \mu \mathrm{g} \mathrm{ml}^{-1} \mathrm{BSA}$ ) [11]. The DNA-protein complexes that formed were separated by $8 \%$ PAGE in $0.5 \times$ TBE buffer.

\section{Measurement of intracellular cAMP concentrations}

Overnight cultures of E. coli were diluted 100-fold into fresh LB medium. Cells were incubated at $30{ }^{\circ} \mathrm{C}$ with shaking at $200 \mathrm{rpm}$ for $21 \mathrm{~h}$. Two milliliters of cultures were removed and centrifuged. Cells were suspended in $200 \mu \mathrm{l}$ of $0.1 \mathrm{~N} \mathrm{HCl}$ and heated at $100{ }^{\circ} \mathrm{C}$ for 10 min. Each sample was then centrifuged at $12000 \mathrm{rpm}$ for $10 \mathrm{~min}$, and the supernatant fluid was adjusted to $\mathrm{pH} 7.0$ with $0.1 \mathrm{~N} \mathrm{NaOH}$. The supernatant fluid was again centrifuged to remove precipitated material. Intracellular cAMP was then measured using the cAMP EIA kit (Assay designs, Ann Arbor, MI). The average intracellular cAMP concentration was expressed in femtomoles per milligram (wet weight) of cells. All assays were performed in duplicate cultures.

\section{Extracellular AI-2 activity assays}

Overnight cultures of E. coli were diluted 100-fold into fresh LB medium. Cells were incubated at $30{ }^{\circ} \mathrm{C}$ with shaking at $200 \mathrm{rpm}$ in flasks. Two milliliters of cultures were removed at regular intervals for the determination of the $\mathrm{OD}_{600}$ and the preparation of cell-free fluids. Cell-free culture fluids were prepared by centrifugation of the cultures at $12000 \mathrm{rpm}$ for $2 \mathrm{~min}$, followed by filtering cleared supernatants through sterilized $0.22-\mu \mathrm{m}$-pore filters, and the resulting cell-free fluids were stored at $-20{ }^{\circ} \mathrm{C}$.

The AI-2 activity in cell-free $E$. coli fluids was measured using the $V$. harveyi strain $\mathrm{BB} 170$ bioluminescence reporter assay, as described previously $[14,16,48]$. Strain BB170 was grown in AB medium overnight and diluted 1:5000 into fresh AB medium. Parallel 96-well microtiter plates containing $180 \mu \mathrm{l}$ of diluted BB170 cultures and $20 \mu \mathrm{l}$ of cell-free fluids were incubated at $30^{\circ} \mathrm{C}$ with shaking. Measurements of luminescence were conducted hourly with a luminometer, while strain BB170 cell density was determined by spreading dilutions onto solid LM medium and counting colonies after overnight growth at $30{ }^{\circ} \mathrm{C}$. Relative light units (RLU) were defined as light emission [counts $\mathrm{min}^{-1} \mathrm{ml}^{-1} \times 10^{3} / \mathrm{CFU} \mathrm{ml}{ }^{-1}$ ] AI-2 activity is reported as fold induction of RLU over the background obtained when LB medium instead of cell-free culture was added.

\section{$\beta$-Galactosidase assays}

Overnight cultures of $E$. coli were diluted 1:100 into fresh LB medium. The cultures were incubated at $30{ }^{\circ} \mathrm{C}$ with shaking at
$200 \mathrm{rpm}$. The microaerobic environment used for bacterial propagation was created by using $40 \mathrm{ml}$ volumes of LB broth in $60-\mathrm{ml}$ serum bottles capped with butyl rubber stoppers, and the cultures were incubated statically at $30^{\circ} \mathrm{C}$. At different time points during cell growth, aliquots were removed for the determination of the $\mathrm{OD}_{600}$, and $\beta$-galactosidase assays were performed as described previously [52]. $\beta$-Galactosidase activity is expressed in Miller units. All assays were performed in triplicate. The error bars in the graphs indicate the standard deviations.

\section{Acknowledgments}

We thank Robert McLean (Texas State University, USA) for generously providing $E$. coli stain ZK126. We also thank Bonnie L Bassler (Princeton University, USA) and Shiyun Chen (Chinese Academy of Sciences, China) for kindly providing $V$. harveyi $\mathrm{BB} 170$. We are grateful to Coli Genetic Stock Center and National Institute of Genetics in Japan for generously providing the other strains and plasmids used in the study. This work was supported by the National Natural Science Foundation of China (50738006) and the One Hundred Talent Project of the Chinese Academy of Sciences.

\section{References}

1 Vendeville A, Winzer K, Heurlier K, Tang CM, Hardie KR. Making 'sense' of metabolism: autoinducer-2, LuxS and pathogenic bacteria. Nat Rev Microbiol 2005; 3:383-396.

2 Fuqua C, Greenberg EP. Listening on bacteria: acyl-homoserine lactone signalling. Nat Rev Mol Cell Bio 2002; 3:685-695.

3 Waters CM, Bassler BL. Quorum sensing: cell-to-cell communication in bacteria. Annu Rev Cell Dev Biol 2005; 21:319-346.

4 Davies DG, Parsek MR, Pearson JP, Iglewski BH, Costerton JW, Greenberg EP. The involvement of cell-to-cell signals in the development of a bacterial biofilm. Science 1998; 280:295298.

5 Ahmer BMM. Cell-to-cell signalling in Escherichia coli and Salmonella enterica. Mol Microbiol 2004; 52:933-945.

6 Sitnikov DM, Schineller JB, Baldwin TO. Control of cell division in Escherichia coli: regulation of transcription of $f t s Q A$ involves both rpoS and SdiA-mediated autoinduction. Proc Natl Acad Sci USA 1996; 93:336-341.

7 Michael B, Smith JN, Swift S, Heffron F, Ahmer BMM. SdiA of Salmonella enterica is a LuxR homolog that detects mixed microbial communities. J Bacteriol 2001; 183:5733-5742.

8 Yao Y, Martinez-Yamout MA, Dickerson TJ, Brogan AP, Wright PE, Dyson HJ. Structure of the Escherichia coli quorum sensing protein SdiA: activation of the folding switch by acyl homoserine lactones. J Mol Biol 2006; 355:262-273.

9 Houdt R, Aertsen A, Moons P, Vanoirbeek K, Michiels CW. Nacyl-1-homoserine lactone signal interception by Escherichia coli. FEMS Microbiol Lett 2006; 256:83-89.

10 Lee J, Jayaraman A, Wood TK. Indole is an inter-species biofilm signal mediated by SdiA. BMC Microbiol 2007; 7:42.

11 Yamamoto K, Yata K, Fujita N, Ishihama A. Novel mode of transcription regulation by SdiA, an Escherichia coli homologue of the quorum-sensing regulator. Mol Microbiol 2001; 41:1187- 
1198.

12 Wei Y, Lee J-M, Smulski DR, Larossa RA. Global impact of $s$ diA amplification revealed by comprehensive gene expression profiling of Escherichia coli. J Bacteriol 2001; 183:2265-2272.

13 Rahmati S, Yang S, Davidson AL, Zechiedrich EL. Control of the AcrAB multidrug efflux pump by quorum-sensing regulator SdiA. Mol Microbiol 2002; 43:677-685.

14 Wang L, Hashimoto Y, Tsao C-Y, Valdes JJ, Bentley WE. Cyclic AMP (cAMP) and cAMP receptor protein influence both synthesis and uptake of extracellular autoinducer 2 in Escherichia coli. J Bacteriol 2005; 187:2066-2076.

15 Wang L, Li J, March JC, Valdes JJ, Bentley WE. luxS-dependent gene regulation in Escherichia coli $\mathrm{K}-12$ revealed by genomic expression profiling. J Bacteriol 2005; 187:8350-8360.

16 Taga ME, Miller ST, Bassler BL. Lsr-mediated transport and processing of AI-2 in Salmonella typhimurium. Mol Microbiol 2003; 50:1411-1427.

17 Xavier K, Miller S, Lu W, et al. Phosphorylation and processing of the quorum-sensing molecule autoinducer-2 in enteric bacteria. ACS Chem Biol 2007; 2:128-136.

18 Li J, Attila C, Wang L, Wood TK, Valdes JJ, Bentley WE. Quorum sensing in Escherichia coli is signaled by AI-2/LsrR: effects on small RNA and biofilm architecture. J Bacteriol 2007; 189:60116020.

19 Chan C, Paul R, Samoray D, et al. Structural basis of activity and allosteric control of diguanylate cyclase. Proc Natl Acad Sci USA 2004; 101:17084-17089.

20 Jenal U, Malone J. Mechanisms of cyclic-di-GMP signaling in bacteria. Annu Rev Genet 2006; 40:385-407.

21 Tamayo R, Tischler AD, Camilli A. The EAL domain protein VieA is a cyclic diguanylate phosphodiesterase. J Biol Chem 2005; 280:33324-33330.

22 Ryjenkov DA, Tarutina M, Moskvin OV, Gomelsky M. Cyclic diguanylate is a ubiquitous signaling molecule in bacteria: insights into biochemistry of the GGDEF protein domain. $J$ Bacteriol 2005; 187:1792-1798.

23 Ryan RP, Fouhy Y, Lucey JF, et al. Cell-cell signaling in Xanthomonas campestris involves an HD-GYP domain protein that functions in cyclic di-GMP turnover. Proc Natl Acad Sci USA 2006; 103:6712-6717.

24 Christen M, Christen B, Folcher M, Schauerte A, Jenal U. Identification and characterization of a cyclic di-GMP-specific phosphodiesterase and its allosteric control by GTP. J Biol Chem 2005; 280:30829-30837.

25 Romling U, Gomelsky M, Galperin MY. C-di-GMP: the dawning of a novel bacterial signalling system. Mol Microbiol 2005; 57:629-639.

26 Galperin MY, Nikolskaya AN, Koonin EV. Novel domains of the prokaryotic two-component signal transduction systems. FEMS Microbiol Lett 2001; 203:11-21.

27 Hisert KB, MacCoss M, Shiloh MU, et al. A glutamate-alanineleucine (EAL) domain protein of Salmonella controls bacterial survival in mice, antioxidant defence and killing of macrophages: role of cyclic diGMP. Mol Microbiol 2005; 56:1234-1245.

28 Henke JM, Bassler BL. Three parallel quorum-sensing systems regulate gene expression in Vibrio harveyi. J Bacteriol 2004; 186:6902-6914.

29 Lenz DH, Mok KC, Lilley BN, Kulkami Rv, Wingreen NS, Bassler BL. The small RNA chaperone Hfq and multiple small
RNAs control quorum sensing in Vibrio harveyi and Vibrio cholerae. Cell 2004; 118:69-82.

30 Miller MB, Skorupski K, Lenz DH, Taylor RK, Bassler BL. Parallel quorum sensing systems converge to regulate virulence in Vibrio cholerae. Cell 2002; 110:303-314.

31 Kanamaru K, Kanamaru K, Tatsuno I, Tobe T, Sasakawa C. SdiA, an Escherichia coli homologue of quorum-sensing regulators, controls the expression of virulence factors in enterohaemorrhagic Escherichia coli O157:H7. Mol Microbiol 2000; 38:805816.

32 Gonzalez Barrios AF, Zuo R, Hashimoto Y, Yang L, Bentley WE, Wood TK. Autoinducer 2 controls biofilm formation in Escherichia coli through a novel motility quorum-sensing regulator (MqsR, B3022). J Bacteriol 2006; 188:305-316.

33 Garcia-Lara J, Shang LH, Rothfield LI. An extracellular factor regulates expression of $s d i A$, a transcriptional activator of cell division genes in Escherichia coli. J Bacteriol 1996; 178:27422748.

34 Ahmer BMM, Reeuwijk JV, Timmers CD, Valentine PJ, Heffron F. Salmonella typhimurium encodes an SdiA homolog, a putative quorum sensor of the LuxR family, that regulates genes on the virulence plasmid. J Bacteriol 1998; 180:1185-1193.

35 Yao Y, Martinez-Yamout MA, Dyson HJ. Letter to the editor: backbone and side chain ${ }^{1} \mathrm{H},{ }^{13} \mathrm{C}$ and ${ }^{15} \mathrm{~N}$ assignments for Escherichia coli SdiA1-171, the autoinducer-binding domain of a quorum sensing protein. J Biomol NMR 2005; 31:373-374.

36 Camilli A, Bassler BL. Bacterial small-molecule signaling pathways. Science 2006; 311:1113-1116.

37 Kovacikova G, Lin W, Skorupski K. Dual regulation of genes involved in acetoin biosynthesis and motility/biofilm formation by the virulence activator AphA and the acetate-responsive LysR-type regulator AlsR in Vibrio cholerae. Mol Microbiol 2005; 57:420-433.

38 Johnson MR, Montero CI, Conners SB, Shockley KR, Bridger SL, Kelly RM. Population density-dependent regulation of exopolysaccharide formation in the hyperthermophilic bacterium Thermotoga maritima. Mol Microbiol 2005; 55:664-674.

39 Tischler AD, Camilli A. Cyclic diguanylate (c-di-GMP) regulates Vibrio cholerae biofilm formation. Mol Microbiol 2004; 53:857869.

40 Weber H, Pesavento C, Possling A, Tischendorf G, Hengge R. Cyclic-di-GMP-mediated signalling within the $\sigma^{\mathrm{S}}$ network of Escherichia coli. Mol Microbiol 2006; 62:1014-1034.

41 Romling U, Amikam D. Cyclic di-GMP as a second messenger. Curr Opin Microbiol 2006; 9:218-228.

42 Deutscher J, Francke C, Postma PW. How phosphotransferase system-related protein phosphorylation regulates carbohydrate metabolism in bacteria. Microbiol Mol Biol Rev 2006; 70:9391031.

43 Inada $\mathrm{T}$, Takahashi $\mathrm{H}$, Mizuno $\mathrm{T}$, Aiba $\mathrm{H}$. Down regulation of cAMP production by cAMP receptor protein in Escherichia coli: an assessment of the contributions of transcriptional and posttranscriptional control of adenylate cyclase. Mol Gen Genet 1996; 253:198-204.

44 Park Y-H, Lee BR, Seok Y-J, Peterkofsky A. In vitro reconstitution of catabolite repression in Escherichia coli. J Biol Chem 2006; 281:6448-6454.

45 Imamura R, Yamanaka K, Ogura T, et al. Identification of the cpdA gene encoding cyclic 3',5'-adenosine monophosphate 
phosphodiesterase in Escherichia coli. J Biol Chem 1996; 271:25423-25429.

46 Mendez-Ortiz MM, Hyodo M, Hayakawa Y, Membrillo-Hernandez J. Genome-wide transcriptional profile of Escherichia coli in response to high levels of the second messenger 3',5'-cyclic diguanylic acid. $J$ Biol Chem 2006; 281:8090-8099.

47 Connell N, Han Z, Moreno F, Kolter R. An E. coli promoter induced by the cessation of growth. Mol Microbiol 1987; 1:195201.

48 Bassler BL, Wright M, Silverman MR. Multiple signalling systems controlling expression of luminescence in Vibrio harveyi: sequence and function of genes encoding a second sensory pathway. Mol Microbiol 1994; 13:273-286.
49 Sambrook J, Russell D, eds. Molecular Cloning: A Laboratory Manual. 3rd Edition, New York: Cold Spring Harbor Laboratory Press, 2001.

50 Koop AH, Hartley ME, Bourgeois S. A low-copy-number vector utilizing beta-galactosidase for the analysis of gene control elements. Gene 1987; 52:245-256.

51 Datsenko KA, Wanner BL. One-step inactivation of chromosomal genes in Escherichia coli K-12 using PCR products. Proc Natl Acad Sci USA 2000; 97:6640-6645.

52 Gerhardt P, Murray RGE, Wood WA, Krieg NR, eds. Methods for General and Molecular Bacteriology. Washington: American Society for Microbiology, 1994. 